\title{
Correction to: Gray Matter Atrophy in the Cortico-Striatal-Thalamic Network and Sensorimotor Network in Relapsing-Remitting and Primary Progressive Multiple Sclerosis
}

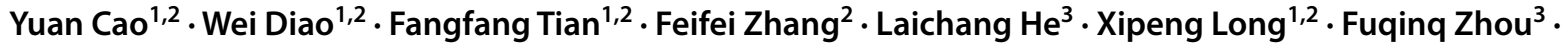 \\ Zhiyun $\mathrm{Jia}^{1,2}$ (1)
}

Published online: 8 April 2021

๑) Springer Science+Business Media, LLC, part of Springer Nature 2021

Correction to: Neuropsychology Review

https://doi.org/10.1007/s1 1065-021-09479-3

Unfortunately, an Acknowledgement section was inadvertently included in the paper. The section was deleted.

The original article has been corrected.

Publisher's Note Springer Nature remains neutral with regard to jurisdictional claims in published maps and institutional affiliations.

The original article can be found online at https://doi.org/10.1007/ s11065-021-09479-3.

Zhiyun Jia

zhiyunjia@hotmail.com

Fuqinq Zhou

fq.chou@yahoo.com

1 Department of Nuclear Medicine, West China Hospital of Sichuan University, Chengdu 610041, China

2 Huaxi MR Research Center (HMRRC), Department of Radiology, West China Hospital of Sichuan University, Chengdu 610041, China

3 Department of Radiology, The First Affiliated Hospital of Nanchang University, Nanchang 330001, China 